\title{
QUANTIFYING DRIVER RESPONSE TIMES BASED UPON RESEARCH AND REAL LIFE DATA
}

\author{
Jeffrey W. Muttart \\ Eastern Connecticut State University \\ Department of Psychology \\ Willimantic, Connecticut, USA \\ E-mail:jmuttart@accidentdynamics.com
}

\begin{abstract}
Summary: The purpose of this paper was to build upon previous research, identify the variables that significantly influence driver response times, and to determine the amplitude (constant) of that influence. The goal is that this research will explain why seemingly analogous published studies have come to very different driver response time results. An analogous driver response situation is defined as being in one of four groups: (1) lead vehicles that were stopped or moving slowly, (2) being cut off (when a vehicle changes lanes into the path of the responding driver), (3) path intrusions, or (4) known lights, icons or sounds. It was found that research that measured response times in analogous situations can be used to estimate the mean response time for a particular situation if adjustments are made to account for methodological differences between the studies. Non-analogous studies are poor predictors of driver response (An anticipated light stimulus response cannot accurately predict the response time to a path intrusion or lead vehicle). Mean driver response times can be predicted within $400 \mathrm{~ms}$ without accounting for individual difference. Therefore, external validity can be obtained regardless of the testing method (closed course, simulator or road), as long as the subject is unaware of either the stimulus or the appropriate response. Having a subject respond to multiple events does not (by itself) suggest that drivers will respond significantly faster.
\end{abstract}

\section{INTRODUCTION}

Over 150 years ago, the chronoscope (stop watch) was invented for the primary purpose of measuring how reaction times differ under various circumstances. However, rule of thumb estimates for response times have frequently been cited by researchers without regard to the specific task. Also, studies that measure the influence of an in-vehicle device may measure the response to a light, icon or lead vehicle and then claim that the results generalize to all response situations. Researchers may rely upon rule of thumb estimates due to the fact that there is little, if any, research to tell us what variables have a significant influence on response time and to what extent (its amplitude).

This research will address several substantive and methodology variables and their influence on driver response times. First, substantive and methodology variables were analyzed using ANOVA to determine if they significantly influence the change in driver response times (Muttart, 2001). Levels of each variable were also determined to ensure that each variable was objectively defined numerically, either ordinally or as a ratio term. In the second step, the variables that reached significance were analyzed using stepwise multiple linear regression (SMLR) from 145 previously published and unpublished driver response time studies (Muttart 
2003a, 2003b). In the third step, the equations that were developed using SMLR were compared to the response times of 161 drivers, who were involved in actual crashes or near crashes that were video recorded in the U.S. and Europe (Muttart, 2004a, 2004b). The SMLR equations were found to predict mean response times within $400 \mathrm{~ms}$ (100\% of the time) and individual response times within $600 \mathrm{~ms} 75 \%$ of the time. Step four involved the development of a parallel assessment method that is referred to as "Adjustment to Baseline" (A2B). A2B assessments involve making adjustments to analogous studies (Muttart, 2003b). The A2B method of estimating driver response also provides a parallel assessment to evaluate the reliability of the SMLR equations.

Each step along the way involved an examination of a greater number of variables and a comparison to a greater number of real-life responses. Now with a larger database of real life responses, this paper will once again look back at the influence of the individual variables.

For each of the four scenario groups, SMLR was used to identify the variables that were most significant when included with other variables. The ANOVA with linear regression analysis gives the slope of the individual variable-response timeline when that variable is considered individually. Although SMLR assumed orthogonal relationships, there will usually be some interaction among variables. For instance, at night, a driver may have smaller average eccentricities due to a smaller field being illuminated; there is no significant correlation between nighttime response time and visual eccentricity. To account for the manner in which the variables work together, a comparison was made of the regression constant (slope) for the variables selected in the SMLR analysis and the regression constant for those variables when considered alone. The regression constant was approximately half when considered with other variables, compared to when it was evaluated alone.

Therefore, a series of "adjustments" were developed that can be applied to analogous driver response time results and may also be used to estimate the influence of a distracter upon a driver's response time.

On the basis of the research by Muttart (2003a), driver response times must be grouped based upon the direction from which the hazard emerges. In earlier research, response times could not be predicted if results from non-analogous driver response times were grouped together. However, if response research was grouped based upon where the hazard emerged (an analogous situation), response times could be predicted with reasonable accuracy. Responses to (1) lead vehicles, (2) being cut off, (3) known sounds and lights, and (4) path intrusions must be grouped in separate databases. Therefore, a response to a known light will not be useful in predicting a path intrusion and a response to a lead vehicle will not be useful in predicting the response to a path intrusion (Muttart, 2003a, 2004a).

\section{METHOD}

A database was developed that consisted of coded entries for over 130 published and 6 nonpublished studies that reported driver response times. The reported driver response times from each study and codes for substantive and methodology variables were entered (see Table 1). If the study reported response times in two different scenarios, then two entries were placed into the database; if there were several hundred subjects but only one scenario, then one entry was 
placed into the database. This method was used so the influence of each variable could be examined. Added to the database were the 195 real-life response times objectively coded as indicated in Table 1.

Table 1. Codes for substantive and methodology variables

\begin{tabular}{|c|c|}
\hline Age & Years \\
\hline Anticipation & $\begin{array}{l}\text { 1-Known stimulus \& Response Multiple responses, 2-Known stimulus and response-- } \\
\text { single event, 3- Known stimulus OR response, multiple, 4-Known stimulus or response- } \\
\text { single, 5-Unknown stimulus and response. }\end{array}$ \\
\hline Brake versus Steer & 1-Brake, 2-Steer right, 2-Steer left \\
\hline Crash & 0 -No, 1-Yes \\
\hline Driving Task & 0 -No, 1-Yes \\
\hline Eccentricity & Degrees \\
\hline Experiment Type & 1-Laboratory, 2-Simulator (steering wheel \& brake), 3-Closed course, 4-Road \\
\hline Gender & 1-Male, 2-Female \\
\hline Headway & In seconds \\
\hline Horn & 0-No horn used, 1-Horn used in avoidance \\
\hline $\mathrm{Km} / \mathrm{h}$ & Velocity \\
\hline Lane & 0-Center, 1-Right, 2-Left \\
\hline Lanes Crossed & 1-From the next, 2-Multiple \\
\hline Left/Right & 1-Right, 2-Left \\
\hline Lighting & 0-Day, 1-Dawn or dusk, 2-Night \\
\hline Movement & 0-Stationary or starting from a stop, 1-Moving without stopping \\
\hline NASA TLX & Score on subjective stress quesytionaire \\
\hline Response & 1-Verbal or press button, 2-Brake or steer was only option, 3-Brake or steer, Brake \& Steer \\
\hline Road & 0-Rural, 1-Suburban/Residential, 2-Arterial, 3-Urban, 4-Highway \\
\hline Road Condition & 1-Dry, 2-Wet, 3-Debris, 4-Snow \\
\hline Stimuli & $\begin{array}{l}\text { 1-Mentally responding to one object, 2-Mentally responding to two spatially separate } \\
\text { objects }\end{array}$ \\
\hline Topography & 1-Straight, 2-Curves, cues or intersections \\
\hline Transition & 1-First driver reaction, 2-First driver response (touch the brake), 3-First vehicle response \\
\hline TTC & Time to contact in seconds from perception point \\
\hline Turning & Destination of the responder, 0-Straight, 1-Right, 2-Left \\
\hline Weather & 1-Sunny, 2-Clear, 3-Rain, 4-Fog or snow \\
\hline
\end{tabular}

The database included 1,813 cases that account for over 10,000 driver responses, the dependent variable and 25 independent variables. There were 167 lead-vehicle response entries, 349 cut-off entries, 341 path-intrusion entries, 184 entries involving responses to known lights, sounds or icons, 141 entries for responses to traffic controls, 67 entries for gaps in traffic, and the remaining were miscellaneous responses. 
Linear regression analysis and one-way analysis of variance (ANOVA) was conducted on each variable. The purpose was to identify the regression constant (slope) for each variable and compare that to the regression constant for the same variable (if found significant in the SMLR analysis). The relationship between the two constants was used to modify the regression constants obtained from the one-way ANOVA along with standard MLR so an adjustment could be obtained for each variable (even those that were not selected during the SMLR analysis). Every variable combination was examined in an attempt to find interactions. The adjustments that were developed were applied to several studies to determine if the differences in the reported results of each study could be accounted for using the adjustment variables.

\section{RESULTS}

As demonstrated in Table 2, studies that measure the response to a known light, sound or icon are influenced by different variables and are not similar to a typical real-life response to a traffic hazard. Furthermore, when examining the terms that best predict response time to a known light, sound or icon, the variables selected by SMLR were all methodology variables (Anticipation,

Table 2. The calculated statistical probabilities using one-way ANOVA for 25 of the variables evaluated relative to the influence upon driver response times

\begin{tabular}{|c|c|c|c|c|}
\hline & Lead vehicle & Cut off & Path Intrusion & Known Lts./Icons/Sounds \\
\hline Age & 0.35 & ND & 0.43 & $\begin{array}{c}0.04 \\
\end{array}$ \\
\hline Anticipation & 0.01 & $\mathbf{0 . 0 0}$ & 0.66 & 0.00 \\
\hline Brake v Steer & ND & 0.50 & 0.84 & 0.02 \\
\hline Crash & ND & 0.49 & 0.39 & ND \\
\hline Driving & 0.12 & 0.00 & 0.00 & 0.16 \\
\hline Eccentricity & 0.00 & 0.00 & 0.00 & 0.00 \\
\hline Experiment Type & 0.08 & 0.00 & 0.00 & 0.44 \\
\hline Gender & 0.87 & ND & 0.08 & 0.43 \\
\hline Headway & 0.00 & ND & ND & ND \\
\hline Horn & ND & 0.44 & 0.26 & ND \\
\hline KM/h & 0.03 & 0.70 & 0.15 & 0.09 \\
\hline Lane & 0.31 & 0.02 & 0.64 & ND \\
\hline Lanes Crossed & ND & 0.00 & 0.13 & $\mathrm{ND}$ \\
\hline Left/Right & ND & 0.38 & 0.70 & 0.18 \\
\hline Lighting & 0.00 & 0.00 & 0.00 & 0.14 \\
\hline Movement & ND & ND & 0.08 & 0.00 \\
\hline NASA TLX & 0.09 & ND & ND & 0.32 \\
\hline Response & 0.32 & $\mathrm{ND}$ & 0.02 & 0.84 \\
\hline Road & ND & 0.90 & 0.60 & 0.00 \\
\hline Stimuli & 0.00 & 0.75 & 0.40 & 0.10 \\
\hline Topography & 0.01 & $\mathbf{0 . 0 0}$ & 0.61 & 0.14 \\
\hline Transition & 0.01 & ND & 0.00 & 0.19 \\
\hline TTC & 0.95 & ND & 0.00 & 0.67 \\
\hline Turning & ND & 0.92 & 0.00 & 0.56 \\
\hline Weather & ND & 0.18 & 0.86 & $\mathrm{ND}$ \\
\hline
\end{tabular}

* The numbers in bold were significant in a one-way analysis and those in shaded cells were significant and accepted when using SMLR $(\mathrm{p}<0.05))$ 
Driving task, experiment type and movement). Therefore, when measuring the response to a known light, icon or sound, the study is likely measuring its own methodology, rather than any particular driving skill. Also, note that age reached significance only when responding to known lights, sounds or icons. Lights and sounds may not allow older driver to utilize their conditioned responses and recognition of traffic patterns.

Other variables that were examined included alcohol (BAC \%), and THC ingestion. Although there may be a wealth of data for these drugs regarding their affects on a person's behavior, there is very little research into the effects of alcohol and THC on the performance of drivers when responding to lead vehicles, being cut off or path intrusions. Due to limited data, no significance was found.

There were four methodology variables: experiment type, transition time, anticipation and the driving task. All four reached significance in at least one response scenario. The influence of methodology variables may be a reason why several authors have performed analogous research yet arrived at different results.

In this research, there was no significant difference between the response times for those who were involved in a crash and those who were not. When evaluating an emergency response, all real-life responses involve urgency. Real-life driver response times were underestimated by studies that did not include the mental workload inherent in driving.

Response times increased when the drivers did not know the stimulus or appropriate response (the anticipation term), but then plateau. This suggests that exposing subjects to multiple events may still yield externally valid results, as long as the subjects do not know both the stimulus and appropriate response in subsequent exposures. This was not the case when examining the response to lead vehicles at long headways. In this case, the reason for a longer response time is due to the expectation that the lead vehicle will be traveling at normal highway speeds. Anticipation reached significance for responses to lead vehicles, but that is due to a confounding variable in the data. The confounding variable is the fact that many of the situations involving low anticipation were responses to lead vehicles at intersections that involved response times of approximately 1 second, while many of the long headway situations involved subjects who knew the stimulus and appropriate response.

Of the 161 emergency responses evaluated on video, 106 involved crashes. There was no difference between the responses of those involved in a crash versus those who avoided the crash. Most scenarios that we are examining involve a great deal of urgency. Based upon the research evaluated, there was no significance between time-to-contact (TTC) and driver response time up until TTCs of 5 seconds, after which time response times increased significantly in path intrusion situations. As expected, if TTC were not a significant influence on response time, speed would not be either. Only when responding to a lead vehicle was speed a significant influence on response time, and in that scenario response time increased at a rate of only $80 \mathrm{~ms}$ for every 10 $\mathrm{km} / \mathrm{h}(6.2 \mathrm{mph})$. Therefore, speed, as a single variable is not an influence upon response time. However, in one of the two significant interactions found, when a driver was engaged in a distracting task, response times increased at a quadratic rate as speed increased (see Figure 1). The other interaction is headway versus topography, in that response time remains constant 
regardless of the headway at curves and intersections, yet increases dramatically at longer headways on straight roads.

The number of objects (stimuli) being mentally processed was a significant influence on response time. When being cut off, it was not significant suggesting that being cut off may involve greater intensity or notice than other stimuli. Also, when a driver in an outside lane was being cut off, particularly the left lane, response times were higher than when in the center lane. Overall, response time increased an average of $300 \mathrm{~ms}$ for eccentricities over 10 degrees in path intrusions and approximately $1 / 4$ second for every 10 degrees when responding to a lead vehicle. When being cut off, the number of lanes away that the intruding vehicle started reached significance, but number of lanes was not a significant influence in any other scenario. However, drivers responded nearly $1 / 5^{\text {th }}$ of a second faster if a path intruding vehicle continued into the responder's path without starting from a stop (or being stationary). Please refer to Table 3 for the amplitude of the adjustments (regression constants).

When responding to illuminated lead vehicle daylight versus darkness had a significant but marginal effect upon driver response time. When responding to a path intrusion, response times increased an average of approximately $1 / 4$ second at night. There are several situations in which an object at night may need much greater time before perception is possible.

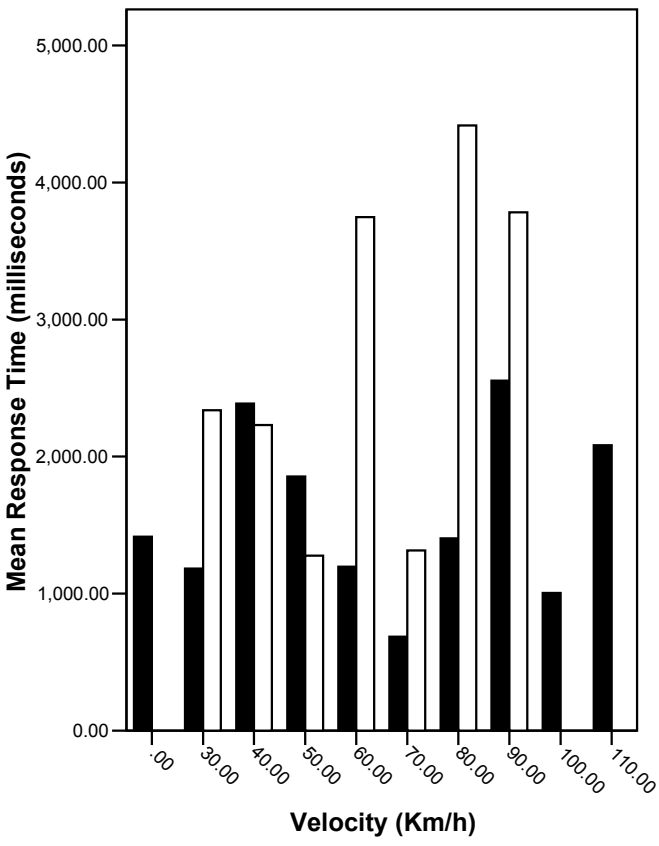

Figure 1. The relationship of speed and driver response time to a lead vehicle. The white bars represent multiple stimuli responses and the black bars single stimulus responses.

Topography could be referred to as a context term. Drivers tend to respond faster in response to lead vehicles or intruding objects when at an intersection or when approaching a curve, compared to when responding to a similar situation on a straight road.

In the second part of this study, the regression constants were used as adjustments to determine if the reported results of each study can be accounted for with substantive and methodology variable adjustments. In Table 4, you can see the adjustments calculated for path intrusions. "Mock" refers to the mock scenario we are attempting to predict response time for. The actual average perception-response time of all real-life drivers who were traveling straight through an intersection and responded to a path intrusion that started from a stop in daylight was 1.3 seconds. Barrett, Kobayashi and Fox (1968), Lechner \& Malaterre (1991), Olson and Sivak (1986), Phelps and Dunn (2000), and SATAI (1999) refer to path intrusion studies. The second row from the bottom shows the reported result of that study and the total adjustments. The bottom row shows the adjusted response time for the mock scenario. 
Table 3. The amplitude of the regression constants of the independent variables.

\begin{tabular}{|l|r|r|r|c|}
\hline & $\begin{array}{c}\text { Lead } \\
\text { vehicle }\end{array}$ & $\begin{array}{c}\text { Cut } \\
\text { off }\end{array}$ & $\begin{array}{c}\text { Path } \\
\text { intrusion }\end{array}$ & $\begin{array}{c}\text { Known Lts. } \\
\text { /Icons/Sounds }\end{array}$ \\
\hline Age & & & & 7.2 \\
\hline Anticipation & & 177 & & 251 \\
\hline Driving Task & -740 & -91 & -350 & -376 \\
\hline Eccentricity & 23 & 11 & 30 & 32 \\
\hline Experiment Type & 81 & 172 & 108 & 252 \\
\hline Headway & 297 & & & \\
\hline KM/h & 8 & & & \\
\hline Lane & & 64 & & \\
\hline Lanes Crossed & & 393 & & \\
\hline Lighting & 98 & 213 & 125 & \\
\hline Movement & & & -164 & -177 \\
\hline Response & & & 109 & \\
\hline Road & & & & -79 \\
\hline Stimuli & 806 & & 802 & \\
\hline Topography & -639 & & -692 & \\
\hline Transition & 350 & 344 & 170 & \\
\hline TTC & & & 103 & \\
\hline Turning & & & 261 & \\
\hline
\end{tabular}

Table 4. The results and methodology of five path intrusion studies in coded form with adjustments for several variables

\begin{tabular}{|l|c|r|r|r|r|r|r|r|r|r|r|r|}
\hline & $\begin{array}{c}\text { Path } \\
\text { Intrusion }\end{array}$ & Mock & Barrett & & Lechner & & Olson & & Phelps & & SATAI & \\
\hline Driving Task & 350 & 1 & 1 & 0 & 1 & 0 & 1 & 0 & 0 & 350 & 1 & 0 \\
\hline Eccentricity & 30 & 5 & 9 & -120 & 5 & 0 & 5 & 0 & 5 & 0 & 5 & 0 \\
\hline Experiment Type & 108 & 4 & 2 & 216 & 2 & 216 & 3 & 108 & 1 & 324 & 3 & 108 \\
\hline Lighting & 125 & 1 & 1 & 0 & 1 & 0 & 1 & 0 & 1 & 0 & 1 & 0 \\
\hline Movement & -164 & 0 & 0 & 0 & 0 & 0 & 0 & 0 & 0 & 0 & 0 & 0 \\
\hline Response & 109 & 3 & 3 & 0 & 3 & 0 & 3 & 0 & 1 & 218 & 3 & 0 \\
\hline Stimuli & 802 & 1 & 1 & 0 & 1 & 0 & 1 & 0 & 1 & 0 & 1 & 0 \\
\hline Topography & -692 & 2 & 1 & -692 & 2 & 0 & 2 & 0 & 1 & -692 & 1 & -692 \\
\hline Transition & 170 & 3 & 1 & 340 & 1 & 340 & 2 & 170 & 1 & 340 & 3 & 0 \\
\hline TTC if $<5$ sec & 103 & 4 & 4 & 0 & 4 & 0 & 4 & 0 & 4 & 0 & 4 & 0 \\
\hline Turning & 261 & 0 & 0 & 0 & 0 & 0 & 0 & 0 & 0 & 0 & 0 & 0 \\
\hline Result/Adjustment & & & 1498 & -256 & 850 & 556 & 1080 & 278 & 635 & 540 & 1901 & -584 \\
\hline Adjusted RT & Actual $=$ & $\mathbf{1 . 3}$ & $\mathbf{1 . 2}$ & & $\mathbf{1 . 4}$ & & $\mathbf{1 . 4}$ & & $\mathbf{1 . 2}$ & & $\mathbf{1 . 3}$ & \\
\hline
\end{tabular}

\section{DISCUSSION}

Some studies measured driver response time from perception up until first reaction (taking the foot off the throttle or first hand movement). Others measured response time to first response or brake reaction time (up until the foot touches the brake or when steering is accomplished). Still 
others measured response time up until full braking or first lateral movement (which accounts for the vehicle latency). Due to a poor vocabulary in the field, all three scenarios have been interchangeably referred to as perception-reaction, perception-response and brake-response time. For clarification, perception-reaction should be when the foot comes off the throttle because that is the driver's first reaction. Brake response time should be the time up to brake application and perception-response should be from perception as an immediate hazard up until first vehicle response.

A change in the stimulus not only brings about a likely different response, but the variables that influence a driver's response have various influences in the various driver response scenarios as well. Mean driver response times may be estimated with a reasonable degree of accuray without accounting for individual characteristics if the direction from which the hazard emerged can be accounted for. This research was based upon how drivers have performed in various research settings and in real-life situations. The results are limited to situations involving an easily identifiable and immediate hazard.

There were four experiment types (laboratory, simulator, closed course and road). A simulator was defined as any response that offered a steering wheel and brake. Therefore, there is a huge disparity in what is considered a "simulator." Subsequent examination of the data has shown that high fidelity simulators such as those at the University of Iowa, GE and others of similar complexity produce results that are very consistent with a "road" study, which is consistent with the findings of McGehee, Mazzae \& Baldwin (2000).

These driver response times did not have a significantly skewed distribution if analogous responses were compared and the methodology of the test is not overly simplified so as to involve a human response time limitation. For those situations when the average response time is near 1 second or less, there will likely be a skew in the distribution because we start to approach a human response limitation.

Drivers' response times are approximately $200 \mathrm{~ms}$ faster when the intruder moves into the responder's path without stopping. Essentially, this means that drivers are recognizing the hazard when the intruder is approximately $200 \mathrm{~ms}$ before the stop line. Speed of the intruder did not influence the response time, which supports a contention that drivers did not calculate stopping distances of an intruder, but most likely judge safety by proximity of other traffic.

When evaluating the response of a driver, we are usually examining the performance of drivers when exposed to one of the most stressful events they will ever face. Urgency may play a role in the response of drivers in less threatening situations, but time-to-contact (TTC)was not significant in this research until TTC in path intrusion situations is greater than 5 seconds. When TTC is greater than 5 seconds, the hazard is becoming less and less of an immediate emergency. This research is examining emergency response times.

Eccentricity had a lesser amplitude when responding to lead vehicles then when responding to path intrusions. This is consistent with a premise that if drivers are mentally occupied by a distraction, they are more likely to fixate in the area of the lead vehicle. It is also noteworthy that steering and braking response times were not significantly different. A steering response does not involve leg movement, but apparently there is a greater cognitive component when steering. 
There is still much we do not know and cannot predict based upon the current data. More research should be done regarding the use of alcohol and distracting stimuli. There is also interest in further examination of the types of eccentricities (up and down, etc.), and separating out the influence of horizontal and vertical curves from warnings. There also appears to be evidence that short following headways may adversely affect response times to path intrusions. There is still a great deal of research required to identify the factors that influence search patterns and driver attention in various situations.

\section{ACKNOWLEDGMENTS}

I would like to thank the Traffic Response \& Safety Research Group for their review of this paper and Eastern Connecticut State University's Psychology and CIT departments and Dana Moffett for his review of the paper.

\section{REFERENCES}

Barrett, G., Kobayashi, M., \& Fox, B. H. (1968). Feasibility of studying driver reaction to sudden pedestrian emergencies in an automobile simulator. Human Factors, 10, 19-26.

Lechner, D., \& Malaterre, G. (1991). Emergency maneuver experimentation using a driving simulator (SAE Paper No. 910016). Warrendale, PA: Society of Automotive Engineers.

McGehee, D. V., Mazzae, E. N., \& Baldwin, G. H. S. (2000). Driver reaction time in crash avoidance research: validation of a driving simulator study on a test track. Proceedings of the International Ergonomics Association 2000 Conference.

Muttart, J. W. (2001). Evaluation of the influence of several variables upon driver perception response times. York, England: Proceedings of the 5th International Conference of the Institute of Traffic Accident Investigators, 116-129.

Muttart, J. W. (2003). Development and Evaluation of Driver Response Time Predictors Based upon Meta Analysis (SAE paper No. 2003-01-0885). Warrendale, PA: Society of Automotive Engineers.

Muttart, J. W. (2003). Evaluation of Methods for Estimating Driver Response Times. Proceedings of the 6th International Conference of the Institute of Traffic Accident Investigators, Stratford-upon-Avon, England.

Muttart, J. W. (2004). DRIVE3: A simplified method for estimating driver response. Auckland, NZ: Australasian and South Pacific Association of Crash Investigators 2004 Conference Proceedings.

Muttart, J. W. (2004). Estimating driver response times. In Handbook for Forensic Human Factors in Litigation (Ch.14). Boca Raton, FL: Taylor \& Francis.

Olson, P. L., \& Sivak, M. (1986). Perception-response time to unexpected roadway hazards. Human Factors, 28, 91-96.

Phelps N.R. and Dunne M.C.M. (2000). Static or kinetic tests, which are influenced most by age? Investigative Ophthalmology and Visual Science, 41(4) S433. 